\title{
HYPONORMAL OPERATORS AND SPECTRAL DENSITY \\ BY
}

J. G. STAMPFLI

Introduction. We say an operator $T$ on a Hilbert space $H$ is hyponormal if $\|T x\| \geqq\left\|T^{*} x\right\|$ for $x \in H$, or equivalently $T^{*} T-T T^{*} \geqq 0$. In this paper we will first examine some general properties of hyponormal operators. Then we restrict our interest to hyponormal operators with "thin" spectra. The importance of the topological nature of the spectrum is evident in our main result (Theorem 4) which states that a hyponormal operator whose spectrum lies on a smooth Jordan arc is normal. We continue with a general discussion of a certain growth condition on the resolvent which obtains for hyponormal operators. We conclude with a counterexample to a relation between hyponormal and subnormal operators. The reader is advised that additional facts about hyponormal operators may be found in [11].

We shall denote the spectrum and the resolvent set of an operator by $\sigma(T)$ and $\rho(T)$, respectively. The spectral radius $R_{\mathrm{sp}}(T)=\sup \{|z|: z \in \sigma(T)\}$. The numerical range $=$ closure $\{z: z=(T x, x)\|x\|=1\}$ is designated by $W(T)$. Throughout the paper the underlying vector space is always a separable Hilbert space $H$.

I. Lemma 1. If $T$ is hyponormal and $(T-z I)^{-1}$ exists (as a bounded operator) then $(T-z I)^{-1}$ is hyponormal.

Proof. Since hyponormality is preserved under translation (see [11, Lemma 1]), we may assume $z=0$. Thus $T^{*} T-T T^{*} \geqq 0$ and hence

$$
0 \leqq T^{-1}\left(T^{*} T-T T^{*}\right) T^{*-1}=T^{-1} T^{*} T T^{*-1}-I .
$$

Now since $A \geqq I$ implies $A^{-1} \leqq I$ we have

$$
I-T^{*} T^{-1} T^{*-1} T \geqq 0,
$$

and hence $\left(T^{*-1} T^{-1}-T^{-1} T^{*-1}\right)=T^{*-1}\left(I-T^{*} T^{-1} T^{*-1} T\right) T^{-1} \geqq 0$ which completes the proof.

THEOREM 1. Let $T$ be hyponormal with $z \in \rho(T)$. Then

$$
\left\|(T-z I)^{-1} x\right\| \leqq 1 / d(z, \sigma(T))
$$

or, equivalently, $\|(T-z I) x\| \geqq d(z, \sigma(T))$ where $\|x\|=1, \quad x \in H$ and $d(z, \sigma(T))=\min \{|z-w|: w \in \sigma(T)\}$.

Received by the editors October 16, 1963. 
Proof. We recall that for $T$ hyponormal, $R_{\mathrm{sp}}(T)^{:}=\|T\|$. Thus for $z \in \rho(T)$, $\|x\|=1$, we have

$$
\begin{aligned}
\left\|(T-z I)^{-1} x\right\| \leqq\left\|(T-z I)^{-1}\right\| & =\max \left\{|w|: w \in \sigma\left[(T-z I)^{-1}\right]\right\} \\
& =1 / \min \{|w|: w \in \sigma[(T-z I)]\} \\
& =1 / \min \{|w-z|: w \in \sigma(T)\}=1 / d(z, \sigma(T)) .
\end{aligned}
$$

The relation $\|(T-z I) x\| \geqq d(z, \sigma(T))$ is now obvious.

It will be convenient to refer to the conclusion of the above theorem by stating that $T$ satisfies condition $G_{1}$; i.e. the resolvent of $T$ has exactly first order rate of growth with respect to the spectrum of $T$.

We note that the resolvent of any operator satisfies a first order rate of growth with respect to its numerical range, i.e.:

REMARK. For any operator $T$,

$$
\|(T-z I) x\| \geqq d(z, W(T)), \quad\|x\|=1 .
$$

Proof. $\|(T-z I) x\| \geqq|((T-z I) x, x)|=|(T x, x)-z| \geqq \min \{|-z|: w \in W(T)\}$. It is well known that, for any operator $T$ on a Hilbert space, $W(T) \supseteq \Sigma(T)$, the convex hull of $\sigma(T)$, and that equality does not hold in general.

TheOREM. 2. If $T$ is hyponormal then $W(T)=\Sigma(T)$.

Proof. In view of the above comment we need only prove $W(T) \subseteq \Sigma(T)$. Let $L$ be a support line for $\Sigma(T)$. Since hyponormality is preserved under translation and multiplication by scalars, we mayassume $L$ is the imaginary axis and $\Sigma(T)$ lies in the left half plane. We now need only show that $a \leqq 0$ for $a+b i \in W(T)$. If not there exists $x \in H,\|x\|=1$ and $T x=(a+b i) x+y$, $(x, y)=0, a>0$. Thus for $c>0$, we have by Theorem 1 ,

$$
c^{2} \leqq\|(T-c I) x\|^{2}=(a-c)^{2}+b^{2}+\|y\|^{2} \text { or } 2 a c \leqq a^{2}+b^{2}+\|y\|^{2}
$$

which is absurd for $c$ approaching $\infty$.

II. In this section we will be concerned only with operators $T$ where $\sigma(T)$ lies on a simple closed rectifiable smooth curve. This, of course, does not imply the spectrum separates the plane. By smooth, we understand the curve to have a continuous second derivative at every point when parametrized with respect to arc length. The curve may then be imbedded in a one-parameterfamily of simple closed rectifiable curves. More explicitly, if $z=g(s), a \leqq s \leqq b$, is the parametrization of the given curve with respect to arc length, then

$$
\begin{aligned}
& f_{1}(\delta)=g(s)+\delta i g^{\prime}(s), \quad 0<\delta<1, \\
& f_{2}(\delta)=g(s)-\delta i g^{\prime}(s)
\end{aligned}
$$

yields the desired one-parameter family. We shall refer to a simple closed rectifiable 
smooth curve simply as an arc. The one-parameter imbedding will be used in the proof of Lemma 2.

We now wish to extend the notion of resolvent set and spectrum. Let $x \in H$, then $(T-z I)^{-1} x=R(z, x)$ is an analytic vector-valued function for $z \in \rho(T)$. A vector-valued function $f(z)$ is called an analytic extension of $R(z, x)$ if it is defined and analytic on an open set $D(f)$ containing $\rho(T)$ and if $(T-z I) f(z)=x$ for $z \in D(f)$. It is very important for us to know $R(z, x)$ possesses only single-valued extensions, i.e., any two extensions coincide on the common domain. Since we are only interested in operators $T$ where $\sigma(T)$ is an arc and is thus nowhere dense in the plane we may conclude that this is always the case. We may now define a maximal single-valued extension of $R(z, x)$ by taking the union of all extensions of $R(z, x)$. We shall designate this maximal single-valued extension of $R(z, x)$ by $R_{e}(z, x)$. We now define

$$
\begin{aligned}
& \rho(T, x)=\left\{z: R_{e}(z, x) \text { is analytic at } z\right\} \\
& \sigma(T, x)=[\rho(T, x)]^{\prime}, \text { the complement of } \rho(T, x) .
\end{aligned}
$$

The definitions of $R_{e}(z, x), \rho(T, x), \sigma(T, x)$ are due to Dunford [4]; the reader will also find a discussion of the properties of $\sigma(T, x)$ there.

LEMMA 2. Let $T$ be a hyponormal operator and $\sigma(T)$ an arc, then $\sigma(T, x) \supseteq \overline{\sigma\left(T^{*}, x\right)}$. (The bar indicates complex conjugate.)

Proof. Let $z_{0} \in \rho(T, x)$. Then $R_{e}(z, x)$ is analytic in $J=\left\{z:\left|z-z_{0}\right| \leqq \delta\right\}$ for some $\delta>0$. Thus $\left((T-z I)^{-1} x, y\right)$ is bounded on $J$ for each $y$ and so $\left\|(T-z I)^{-1} x\right\| \leqq K$ for $z \in J$ by the Banach-Steinhaus theorem. Since $(T-z I)^{-1}$ is hyponormal for $z \in J \backslash \sigma(T)$ we may conclude that $\left\|\left(T^{*}-z I\right)^{-1} x\right\| \leqq K$ for $\bar{z} \in J \backslash \sigma(T)$. We know that $\left(T^{*}-z I\right)^{-1} x$ is analytic for $\bar{z} \in J \backslash \sigma(T)$. We now wish to extend $\left(T^{*}-z I\right)^{-1} x$ to be continuous on $\overline{J_{1} \cap \sigma(T)}$ where

$$
J_{1}=\left\{z:\left|z-z_{0}\right|<\delta / 2\right\} \text {. }
$$

By the resolvent equation we have

$$
\begin{aligned}
\left\|\left[\left(T^{*}-z_{1} I\right)^{-1}-\left(T^{*}-z_{2} I\right)^{-1}\right] x\right\| & =\left|z_{1}-z_{2}\right| \cdot\left\|\left(T^{*}-z_{1} I\right)^{-1} x\right\| \cdot\left\|\left(T^{*}-z_{2} I\right)^{-1} x\right\| \\
& \leqq\left|z_{1}-z_{2}\right| \cdot K^{2} \text { for } z_{1}, z_{2} \in \overline{J_{1} \cap \rho(T)} .
\end{aligned}
$$

For $w \in J_{1} \cap \sigma(T)$ we choose a sequence $\left\{z_{i}\right\}, z_{i} \in \overline{J_{1} \cap \rho(T)}$ with $z_{i} \rightarrow w$. Since the $z_{i}$ 's form a Cauchy sequence, $\left\{\left(T^{*}-z_{i} I\right)^{-1} x\right\}$ is a Cauchy sequence and we define $\left(T^{*}-w I\right)^{-1} x$ as its limit. Consider the vector-valued function

$$
h(z)=\left(T^{*}-z I\right)^{-1} x
$$

defined on $\overline{J_{1} \cap \sigma(T)}$ by this process. Clearly $h(z)$ is continuous on $\overline{J_{1} \cap \sigma(T)}$ and $\lim _{z \rightarrow z_{1}}\left(T^{*}-z\right)^{-1} x=h\left(z_{1}\right)$ for $z \in \overline{\rho(T)}$. Thus by a well-known theorem (see $[7$, p. 184] $),\left(T^{*}-z I\right)^{-1} x$ may be extended to be analytic in $\overline{J_{1}}$ and so at $\overline{z_{0}}$. Thus 
$z_{0} \in \overline{\rho\left(T^{*}, x\right)}$ and hence $\rho(T, x) \subseteq \overline{\rho\left(T^{*}, x\right)}$. Finally $\sigma(T, x) \supseteq \overline{\sigma\left(T^{*}, x\right)}$ which completes the proof.

Though it might appear to be of little consequence, the last lemma is one of the key steps in the proof of the main theorem. It, in fact, ensures that the resolution of the identity for our operator is countably additive. In general $\sigma(T, x) \notin \overline{\sigma\left(T^{*}, x\right)}$ as the reader may easily verify for himself by considering the operator

$$
\left|\begin{array}{rr}
1 & 1 \\
0 & -1
\end{array}\right|
$$

on a two-dimensional Hilbert space. Among other things, $\sigma(T, x) \supseteq \overline{\sigma\left(T^{*}, x\right)}$, for all $x \in H$,implies the eigenvectors of $T$ corresponding to distinct eigenvalues are perpendicular.

THEOREM 3. If $T$ is hyponormal and $\sigma(T)$ is an arc, then $\sigma(T, x) \cap \sigma(T, y)=0$ implies $(x, y)=0$.

Proof. The function

$$
\left.f(z)=\left((T-z I)^{-1} x, y\right)=\left(x,\left(T^{*}-\bar{z} I\right)^{-1} y\right)=\overline{\left(\left(T^{*}-\bar{z} I\right)^{-1} z, x\right.}\right)
$$

is analytic for $z \notin \sigma(T, x)$ and $z \notin \overline{\sigma\left(T^{*}, y\right)}$. Now since $\overline{\sigma\left(T^{*}, y\right)} \subseteq \sigma(T, y)$, we have $\sigma(T, x) \cap \overline{\sigma\left(T^{*}, y\right)}=0$; hence $f(z)$ is analytic everywhere. Clearly $f(z)$ vanishes at infinity and therefore must be identically zero. However, $f(z)=\sum_{n=0}^{\infty}-\left(T^{n} x, y\right) z^{-(n+1)}$, hence all coefficients of $z^{n}$ must be zero, in particular $(x, y)$. This proof is similar to one by J. Schwartz for self-adjoint operators.

Corollary. If $T$ is hyponormal and $\sigma(T)$ is an arc, then $T$ satisfies Dunford's boundedness condition B. More explicitly, $\sigma(T, x) \cap \sigma(T, y)=0$ implies $\|x\| \leqq K\|x+y\|, K$ constant, for all $x, y \in H$.

It is clear from Theorem 3 that $K$ may be taken as 1 , a fact we will use in the main theorem.

THEOREM 4. If $T$ is hyponormal and $\sigma(T)$ is an arc, then $T$ is normal.

Proof. Under the hypothesis of the theorem, $T$ must satisfy the growth condition $G_{1}$ (Theorem 1) and the boundedness condition $B$ (Corollary to Theorem 3 ). We may thus invoke Theorems 15 and 18 of [5] to conclude that $T$ is a scalar type spectral operator. To show that this scalar operator is normal, one may observe that the $K$ we showed to be 1 is actually the bound of the norms of the projections which constitute the resolution of the identity for our operator. For the reader who prefers not to follow this argument through the proof in question, we recall that any hyponormal scalar operator $T$, where area $[\sigma(T)]=0$, is normal (Theorem 4 of [11]). 
COROLlaRY. If $T$ is hyponormal and $\sigma(T)$ is real, then $T$ is self-adjoint.

COROLlaRY. If $T$ is hyponormal and $\sigma(T)$ lies on the unit circle, then $T$ is unitary.

Definition. An operator $T$ is quasi-normal (see [2]) if $\left(T^{*} T\right) T=T\left(T^{*} T\right)$. An operator $T$ on a Hilbert space $H$ is subnormal if there exists a Hilbert space $K, K \supseteq H$, and a normal operator $B$ defined on $K$ with $T x=B x$ for $x \in H$.

REMARK. One has the following inclusion relation for classes of operators:

Normal $\subseteq$ Quasi-normal $\subseteq$ Subnormal $\subseteq$ Hyponormal.

The inclusions are all proper. It is interesting to note that differing degrees of spectral thinness or density imply normality for these operators.

If $T$ is quasi-normal and $\sigma(T)$ has no interior, then $T$ is normal. (This result does not appear in the literature but it is not difficult to verify.)

If $T$ is subnormal and area $[\sigma(T)]=0$ then $T$ is normal (see[1]).

If $T$ is hyponormal and $\sigma(T)$ is an arc, then $T$ is normal.

III. We would now like to consider the implication of the growth condition $\mathrm{G}_{1}$, i.e., if $\|(T-z I) x\| \geqq d(z, \sigma(T)) \cdot\|x\|$, what can one conclude? We first mention two results which appear in the literature.

TheOREM A (NIEMINEN [9]). If $T$ satisfies condition $\mathrm{G}_{1}$ and $\sigma(T)$ is real, then $T$ is self-adjoint.

TheOREM B (Donaghue [3]). If $T$ satisfies condition $\mathrm{G}_{1}$ and $\sigma(T)$ lies on the unit circle, then $T$ is unitary.

As one might expect, we also have

THeOREM C. If $T$ satisfies condition $\mathrm{G}_{1}$ and $\sigma(T)$ is a finite set of points, then $T$ is normal.

Proof. For $z_{j} \in \sigma(T)$ we choose $R$ small enough to ensure that the points of the circle $\left|z-z_{j}\right|=R$ are far from the rest of $\sigma(T)$. Then

$$
E_{j}=\frac{1}{2 \pi i} \int_{\left|z-z_{j}\right|=R}(T-z I)^{-1} d z
$$

is a projection which commutes with $T$ and

$$
\left\|E_{j}\right\| \leqq \frac{1}{2 \pi} \int_{\left|z-z_{j}\right|=R}\left\|(T-z I)^{-1}\right\| d z \leqq \frac{1}{2 \pi} 2 \pi R \frac{1}{R}=1 .
$$

Thus $E_{j}$ is self-adjoint. Now for $x \in E_{j} H$ we have

$$
\left\|\left(T-z_{j} I\right) x\right\|=\left\|\frac{1}{2 \pi j} \int_{\left|z-z_{j}\right|=R}\left(z-z_{j}\right)\left(T-z_{j} I\right)^{-1} x d z\right\| \leqq R .
$$

Letting $R \rightarrow 0$ we have $T x=z_{j} x$ for $x \in E_{j} H$. Thus $T=\sum_{j=1}^{n} z_{j} E_{j}$ and since the $E_{j}$ 's are self-adjoint, $T$ is normal. 
CoRollary If $T$ satisfies condition $\mathrm{G}_{1}$ and the underlying space is finitedimensional, then $T$ is normal.

However, if $T$ is completely continuous and satisfies condition $G_{1}, T$ need not be normal. We will sketch a simple example to illustrate this. The operator

$$
T_{1}=\left|\begin{array}{ll}
0 & 1 \\
0 & 0
\end{array}\right|
$$

does not satisfy condition $\mathrm{G} 1$ at zero since

$$
\left(T_{1}-z I\right)^{-1}=\left|\begin{array}{ll}
z^{-1} & z^{-2} \\
0 & z^{-1}
\end{array}\right|
$$

We will now define an operator $T_{2}$ in such a manner that $T=T_{1} \oplus T_{2}$ does satisfy condition $G_{1}$ and moreover is completely continuous. Let $\left\{f_{i}\right\}_{i=1}^{\infty}$ be an orthogonal basis for $H_{2}$. We now set $T_{2} f_{i}=a_{i} f_{i}$ where the $a_{i}$ 's are complex numbers placed on circles concentric to the origin with sufficient density to ensure that $\min _{i}\left|z-a_{i}\right| \leqq|z|^{2}$ for each $z, 0<|z|<1$. This can clearly be done with zero as the only limit point of the $a_{i}$ 's. The operator $T=T_{1} \oplus T_{2}$ defined on $H_{1} \oplus H_{2}$ is completely continuous and satisfies condition $G_{1}$ by construction but it is obviously not normal. This example also illustrates that if $T$ satisfies condition $G_{1}$ and $M$ is a reducing subspace of $T$ then $\left.T\right|_{M}$ may not satisfy condition $G_{1}$, a fact which makes life more difficult.

We close this section by posing one problem. If $T$ satisfies condition $G_{1}$ and $\sigma(T)$ is a smooth rectifiable convex arc must $T$ be a normal operator? Since the proof of Theorem 2 uses only the growth condition $G_{1}$ we may conclude that for such a $T, \Sigma(T)=W(T)$. Combining this with [8] we observe that $T$ has no residual spectrum and that the point spectrum of $T$ acts in the correct manner (as that of normal operator) and can be factored out. The problem is thus reduced to considering only those operators with continuous spectra.

Using a technique of Aronszajn, Dunford, and Schwartz (see [10]), we find $T$ has proper invariant subspaces and then there remains the task of piecing these together to form a resolution of the identity.

IV. We will begin this section with a few results on subnormal and hyponormal operators which will be helpful in clarifying a conjectured relation between them.

Lemмa. 3 Let $T$ be hyponormal on $H$; then $\|T x\|=\left\|T^{*} x\right\|$ if and only if $T^{*} T x=T T^{*} x$.

Proof. The proof of the sufficiency is obvious. If $\|T x\|=\left\|T^{*} x\right\|$ then $\left(\left(T^{*} T-T T^{*}\right) x, x\right)=0$ and hence for $y \in H$,

$$
\left|\left(\left(T^{*} T-T T^{*}\right) x, y\right)\right|^{2} \leqq\left|\left(\left(T^{*} T-T T^{*}\right) x, x\right)\right| \cdot\left|\left(\left(T^{*} T-T T^{*}\right) y, y\right)\right|=0
$$

by the generalized Schwarz inequality for positive operators. Since $y$ is arbitrary we have $T^{*} T x=T T^{*} x$ as desired. 
LemmA 4. Let $T$ be hyponormal on $H$ and set $M=\left\{x \in H:\|T x\|=\left\|T^{*} x\right\|\right\}$; then $M$ is a closed subspace of $H$.

Proof. Obvious from Lemma 3.

The subspace $M$ defined above need not be invariant if $T$ is simply hyponormal. However, for subnormal operators, $M$ is invariant as we shall show and this fact is sometimes useful in distinguishing between the two classes.

Lemma 5. If $T$ is subnormal on $H$ and $B$ the normal extension of $T$, then $\|T x\|=\left\|T^{*} x\right\|$ if and only if $B^{*} x=T^{*} x \in H$.

Proof. Since $\left\|T^{*} x\right\|=\|T x\|=\|B x\|=\left\|B^{*} x\right\|$ the proof is obvious for $\|T x\|=\left\|T^{*} x\right\|$ and the argument is reversible.

TheOREM 5. If $T$ is subnormal on $H$ with normal extension $B$ and $M=\left\{x \in H:\|T x\|=\left\|T^{*} x\right\|\right\}$, then $M$ is a closed invariant subspace of $T$.

Proof. In view of Lemma 4 we have only to prove $M$ is invariant, that is, $\left\|T x^{2}\right\|=\left\|T^{*} T x\right\|$ for $x \in M$. From Lemma 5 we know $T^{*} x=B^{*} x \in H$ for $x \in M$ and hence

$\left\|T^{2} x\right\|=\left\|B^{2} x\right\|=\left\|B^{*} B x\right\|=\left\|B B^{*} x\right\|=\left\|B T^{*} x\right\|=\left\|T T^{*} x\right\|=\left\|T^{*} T x\right\|$

which completes the proof.

Corollary. If $T$ is subnormal on $H$ and there exists $x \in H,\|x\|=1$ such that $\|T x\|=\|T\|$, then $T$ has a proper invariant subspace

Proof. Since $\|T x\|=\|T\|$ there exists $y \in H,\|y\|=1$, such that $\left\|T^{*} y\right\|=\|T\|$ (this is true for arbitrary operators). Then

$$
\|T\| \leqq\left\|T^{*} y\right\| \leqq\|T y\| \leqq\|T\|
$$

which implies the subspace $M$ is not empty. Now if $M \neq H$ we are done. If $M=H$, then $T$ is normal, and invariant subspaces abound.

In [6], Halmos exhibited a hyponormal operator whose square was not hyponormal. S. Berberian has asked (mimeographed University of Michigan seminar notes) whether an operator must be subnormal if all its powers are hyponormal. We are now prepared to give a negative answer to that question. Let $\left\{f_{i}\right\}_{1=-\infty}^{\infty}$ be an orthonormal basis for $H$ and define

$$
T f_{i}=\left\{\begin{array}{ll}
f_{i+1}, & i \leqq 0 \\
2 f_{i+1}, & i>0
\end{array} .\right.
$$

Then $T^{k} f_{i}=b_{i, k} f_{i+k}$ where $\left|b_{i, k}\right| \leqq\left|b_{i+1, k}\right|$ so $T^{k}$ is hyponormal for $k=1,2, \cdots$. Since $\left\|T f_{0}\right\|=\left\|T^{*} f_{0}\right\|$ but $\left\|T^{*} T f_{0}\right\| \neq\left\|T^{2} f_{0}\right\|$ we must conclude that $T$ is not subnormal. 


\section{BIBLIOGRA PHY}

1. E. Bishop, Spectral theory for operators on a Banach space, Trans. Amer. Math. Soc. 86 (1957), 414-445.

2. A. Brown, On a class of operators, Proc. Amer. Math. Soc. 4 (1953), 723-728.

3. W. Donaghue, On a problem of Nieminen, Inst. Hautes Études Sci. Publ. Math. 16 (1963), 127-129.

4. N. Dunford, Spectral theory. II. Resolutions of the identity, Pacific J. Math. 2 (1952), 559-614.

5. - A survey of the theory of spectral operators, Bull. Amer. Math. Soc. 64 (1958), 217-274.

6. P. Halmos, Normal dilations and extensions of operators, Summa Brasil. Math. 2 (1950), 125-134.

7. E. Hille, Analytic function theory, Vol. I, Ginn, Boston, Mass., 1959.

8. C. H. Meng, On the numerical range of an operator, Proc. Amer. Math. Soc. 14 (1963), 167-171.

9. T. Nieminen, A condition for the self-adjointness of a linear operator, Ann. Acad. Sci. Fenn. Ser. A I No. 316 (1962), 3-5.

10. J. Schwartz, Subdiagonalization of operators in Hilbert space with compact imaginary part, Comm. Pure Appl. Math. 15 (1962), 159-172.

11. J. Stampfli, Hyponormal operators, Pacific J. Math. 12 (1962), 1453-1458.

NeW YORK UNIVERSITY,

New York, New YoRK 